\title{
Elevated plasma fatty acid-binding protein 3 is related to prolonged corrected QT interval and reduced ejection fraction in patients with stable angina
}

\author{
Yung-Chuan Lu'1,8, Thung-Lip Lee ${ }^{2,8}$, Chin-Feng Hsuan 2,6,12, Wei-Chin Hung2,6, Cheng-Ching Wu ${ }^{2,7,11}$, \\ Chao-Ping Wang2,8, Ching-Ting Wei3,8,9,10, Teng-Hung Yu2,7, Fu-Mei Chung2, Yau-Jiunn Lee5, and I-Ting \\ Tsai ${ }^{4,6}$ \\ 1. Division of Endocrinology and Metabolism, Department of Internal Medicine, E-Da Hospital, Kaohsiung, 82445 Taiwan \\ 2. Division of Cardiology, Department of Internal Medicine, E-Da Hospital, Kaohsiung, 82445 Taiwan. \\ 3. Division of General Surgery, Department of Surgery, E-Da Hospital, Kaohsiung, 82445 Taiwan. \\ 4. Department of Emergency, E-Da Hospital, Kaohsiung, 82445 Taiwan. \\ 5. Lee's Endocrinology Clinic, Pingtung, 90000 Taiwan. \\ 6. School of Medicine, College of Medicine, I-Shou University, Kaohsiung, 82445 Taiwan. \\ 7. The School of Chinese Medicine for Post Baccalaureate, College of Medicine, I-Shou University, Kaohsiung, 82445 Taiwan. \\ 8. School of Medicine for International Students, College of Medicine, I-Shou University, Kaohsiung, 82445 Taiwan. \\ 9. Department of Biomedical Engineering, I-Shou University, Kaohsiung, 82445 Taiwan. \\ 10. Department of Electrical Engineering, I-Shou University, Kaohsiung, 82445 Taiwan. \\ 11. Division of Cardiology, Department of Internal Medicine, E-Da Cancer Hospital, Kaohsiung 82445 Taiwan. \\ 12. Division of Cardiology, Department of Internal Medicine, E-Da Dachang Hospital, Kaohsiung, Taiwan.
}

$\triangle$ Corresponding author: Dr. I-Ting Tsai, E-Da Hospital, I-Shou University, No. 1, Yi-Da Rd, Jiau-Shu Village, Yan-Chao District, Kaohsiung, 82445, Taiwan. Tel: +886-7-615-1100 ext. 5914 or 5018; E-mail: ed100614@edah.org.tw.

(C) The author(s). This is an open access article distributed under the terms of the Creative Commons Attribution License (https://creativecommons.org/licenses/by/4.0/). See http://ivyspring.com/terms for full terms and conditions.

Received: 2020.10.14; Accepted: 2021.03.01; Published: 2021.03.11

\begin{abstract}
Background: Higher concentrations of plasma fatty acid-binding protein 3 (FABP3) play a role in the development of cardiovascular events, cerebrovascular deaths, and acute heart failure. However, little is known about the relationship between plasma FABP3 level and prolonged QT interval and reduced ejection fraction (EF). This study aimed to investigate the relationship between plasma FABP3 level and prolonged corrected QT (QTc) interval and reduced EF in patients with stable angina. Inflammatory cytokine and adipocytokine levels were also measured to investigate their associations with plasma FABP3.

Methods: We evaluated 249 consecutive patients with stable angina. Circulating levels of FABP3 were measured by ELISA. In addition, 12-lead ECG and echocardiography recordings were obtained from each patient.

Results: Multiple regression analysis showed that high-density lipoprotein cholesterol, high sensitivity C-reactive protein (hs-CRP), white blood cell (WBC) count, visfatin, adiponectin, FABP4, heart rate, QTc interval, left atrial diameter, left ventricular mass index, end-systolic volume, end-systolic volume index, fractional shortening, and EF were independently associated with FABP3 (all $p<0.05$ ). Patients with an abnormal QTc interval had a higher median plasma FABP3 level than those with a borderline and normal QTc interval. With increasing FABP3 tertiles, the patients had higher frequencies of abnormal QTc interval, left ventricular systolic dysfunction, and all-cause mortality, incrementally lower EF, higher WBC count, and higher levels of hs-CRP, visfatin, adiponectin, and FABP4.

Conclusion: This study indicates that plasma FABP3 may act as a surrogate parameter of prolonged QTc interval and reduced EF in patients with stable angina, partially through the effects of inflammation or cardiomyocyte injury. Further studies are required to elucidate whether plasma FABP3 plays a role in the pathogenesis of QTc prolongation and reduced EF.
\end{abstract}

Key words: fatty acid-binding protein 3; corrected QT Interval; ejection fraction; inflammation; stable angina 


\section{Introduction}

Cardiovascular disease (CVD) is one of the main causes of death in most countries [1] and includes related heart and blood vessel problems such as coronary heart disease, heart failure, congenital heart disease, raised blood pressure, peripheral artery disease, rheumatic heart disease, and cerebrovascular disease [2]. An estimated 25 million new cases of heart disease are diagnosed each year [3], and thus identifying the risk factors for CVD is of crucial importance to improve long-term therapeutic outcomes and prevent and reduce wasting medical resources.

Chronic heart failure (CHF) is one of the most common cardiovascular disorders. It is associated with unfavorable outcomes and often coexists with other chronic diseases that further affect the prognosis [4,5]. Among a variety of parameters reflecting disease severity, left ventricular ejection fraction (LVEF) remains one of the strongest long-term prognostic factors.

Prolonged QTC interval and reduced EF are complex traits which can be affected by both genetic and environmental factors [6,7]. Previous studies have shown that during reperfusion of ischemic myocardium, cytokines such as platelet-activating factor generated by activated neutrophils can cause arrhythmia [8,9]. Furthermore, Chung et al. showed that elevated C-reactive protein (CRP) may reflect an inflammatory state that promotes the persistence of atrial fibrillation (AF) [10]. Moreover, ArroyoEspliguero et al. demonstrated an inverse correlation between high-sensitive (hs)-CRP serum concentration and LVEF, and that hs-CRP was an independent predictor of NYHA functional class in patients with chronic stable angina [11]. Moreover, increased levels of inflammatory mediators in patients with stable angina have been associated with cardiac fibrosis, arrhythmias, and CHF $[8,9,12]$, resulting in a higher risk of these disorders and even death.

Fatty acid-binding protein 3 (FABP3) (also known as heart-type fatty acid-binding protein or $\mathrm{H}-\mathrm{FABP}$ ) is a type of intra-cardiac protein that plays an important role in the metabolism of fatty acids inside cardiomyocytes [13]. FABP3 is mainly found in the heart and has a molecular weight of $15 \mathrm{kDa}$. When there is an ischemic injury to the myocardium, FABP3 is rapidly released into the circulatory system and is then eliminated by the kidneys. Consequently, serum FABP3 measurements can reflect myocardial injury. Furthermore, FABP3 has been associated with other clinical parameters in patients with acute myocardial infarction (AMI) such as EF, length of hospital stay, high-sensitive troponin $\mathrm{T}$ and N-terminal pro-brain natriuretic peptide levels as well as inflammatory markers (CRP, leucocytes) [14]. Moreover, FABP3 is not only a sensitive marker of myocardial injury when it is over-expressed, but it has also been shown to promote growth, inflammation, and migration of vascular smooth muscle cells, and thus it could potentially play a role in the pathophysiology of in-stent restenosis [15]. Given the association between QT interval prolongation, reduced $\mathrm{EF}$, coronary artery disease (CAD), and inflammation, and given the inflammatory characteristics of a high plasma FABP3 level and its relationship with CVD, we hypothesized that FABP3 may be a surrogate marker independently associated with QTc interval prolongation and reduced EF in humans.

To the best of our knowledge, little is known about the relationship between FABP3 level and cardiac electro-pathology and LVEF. Therefore, the aim of this study was to investigate whether FABP3 levels were associated with a prolonged QTc interval and reduced EF in a cohort of patients with stable angina. Inflammatory cytokine and adipocytokine levels were also measured to investigate their associations with plasma FABP3.

\section{Methods}

\section{Study participants}

We prospectively screened consecutive patients referred for percutaneous coronary interventions (PCIs) from July 2007 to July 2015 at E-Da Hospital. We included patients with both stable angina pectoris and successful PCI. Stable angina pectoris was diagnosed by the cardiologist in charge as previously described [16,17]. Successful PCI was defined as a final angiographic residual stenosis of $<30 \%$ as determined by quantitative coronary angiography without flow-limiting dissection or occlusion of the large branch $(>1 \mathrm{~mm})$ and a resulting Thrombolysis in Myocardial Infarction grade 3 [18]. The exclusion criteria were patients who experienced periprocedural myocardial injury, defined as an increase in troponin $\mathrm{T}$ three times greater than the upper limit of the reference range 24 hours after PCI [19], patients with a history of acute coronary syndrome (ACS) and psychosis, inflammatory diseases (including infection or sepsis), collagen diseases, liver diseases, malignancy, steroid use, and those with a bundle branch block pattern. In addition, patients with moderate to severe valvular heart disease diagnosed according to a previous report [20], patients taking medications which may have influenced the QT interval including psychotropic medications and class I (e.g. mexiletine, flecainide, quinidine, and procainamide) and class III (e.g. amiodarone, dronedarone, and vernakalant) anti- 
arrhythmic medications were also excluded from the study. Written informed consent was obtained from the patients before enrollment. The study followed the guidelines of and was approved by the Human Research Ethics Committee at our institution.

Before the coronary angiography examinations, detailed records of each patient with regards to personal and medical histories were reviewed. The following CVD risk factors were assessed. Patients with a current or prior diagnosis of type 2 diabetes and those receiving medical therapy for type 2 diabetes were defined as having type 2 diabetes in accordance with the World Health Organization guidelines [21]. Arterial hypertension was diagnosed in patients with resting blood pressure values $\geq 140 / 90 \mathrm{mmHg}$, and in patients with a history of hypertension and taking anti-hypertensive drugs. Hyperlipidemia was defined as a fasting total serum cholesterol level $>200 \mathrm{mg} / \mathrm{dL}$, low-density lipoprotein cholesterol (LDL-C) level $>130 \mathrm{mg} / \mathrm{dL}$, high-density lipoprotein cholesterol (HDL-C) level $<40 \mathrm{mg} / \mathrm{dl}$ or serum triglyceride level $>180 \mathrm{mg} / \mathrm{dL}$; moreover, all patients receiving lipid-lowering therapy, which was routinely administered at our institution during the study period, were diagnosed with hyperlipidemia. Smoking status was classified as non-smoker, former smoker (having stopped smoking for $\geq 1$ year), or current smoker. Current and former smokers were grouped for analysis and compared to the never smokers.

\section{Laboratory measurements}

Before the coronary angiography examinations, plasma biochemical parameters were measured in all of the patients after fasting for 8 hours. All biochemical analyses were performed at the E-Da Hospital laboratory within 2 hours of the blood samples being drawn. Serum triglycerides, total cholesterol, LDL-C, HDL-C, albumin, glucose, and white blood cell (WBC) count were determined using standard commercial methods on a parallelmultichannel analyzer (SYNCHRON, Los Angeles, CA). Hemoglobin A1c (HbA1c) was measured using high performance liquid chromatography. Serum creatinine was analysed according to the kinetic Jaffé method on a SYNCHRON CX System analyzer (SYNCHRON, Los Angeles, CA) using reagents from Beckman (Beckman Coulter Diagnostic, Los Angeles, CA). In addition, the concentrations of plasma FABP3 and FABP4 were determined using enzyme-linked immunosorbent assay kits (Invitrogen, Thermo Fisher Scientific Inc., USA and R\&D Systems Inc., Minneapolis, MN, USA). The dilution and standard curves were parallel, and the intra- and inter-assay coefficients of variation of the assays were $3.9 \%(n=8)$ and $6.2 \%(\mathrm{n}=8)$, respectively, for FABP3, and $3.4 \%$ to $5.8 \%(n=3)$ and $3.1 \%$ to $6.2 \%(n=4)$, respectively, for FABP4. The concentration of plasma visfatin was determined using a commercial enzyme immunoassay kit (Phoenix Pharmaceuticals, Belmont, CA). The detection limit of this assay was $0.1 \mathrm{ng} / \mathrm{mL}$. The serum level of high molecular weight adiponectin was determined using a commercial solid phase ELISA kit (B-Bridge International, Sunnyvale, CA). The dilution curve was parallel to the standard curve. The inter- and intra-assay coefficients of variation of the assay were $3.2-7.3 \%(n=3)$ and $3.1-6.2 \%(n=4)$, respectively. A high-sensitivity method was used to measure levels of plasma CRP with an IMMAGE system (Beckman Coulter, Immunochemistry Systems, Brea, CA, USA) that had a detection limit of $0.2 \mathrm{mg} / \mathrm{L}$. The intra-assay coefficient of variation was $4.2 \%$ to $8.7 \%$ for hs-CRP. Samples were assessed in duplicate in a single experiment. Estimated glomerular filtration rates (eGFRs) were calculated using the CKD-EPI two-concentration race equation [22].

\section{Electrocardiogram, QT and QTc interval measurements}

Twelve-lead ECG was recorded for analysis during the baseline examination using a standardized protocol when the patient was enrolled in the study. At least two cardiologists who were blinded to this study manually measured the QT interval. Full details about the electrocardiogram, QT and QTc interval measurements are provided in the supplementary materials [23-27].

\section{Echocardiographic examination}

All patients received standard echocardiography at the follow-up visit 1 month after the PCI. Standard examinations were performed by the same experienced physician, and all of the echocardiographic measurements followed the recommendations of the American Society of Echocardiography, with at least three cycles being analyzed for each variable $[28,29]$. Full details about the echocardiographic measurements are provided in the supplementary materials [30].

\section{Follow-up}

The patients were reevaluated at 3, 6, and 12 months after discharge and then annually until July 2015. All of the participants received a CVD care program including a general health condition questionnaire and regular blood tests by a trained nurse to determine the occurrence of major adverse cardiovascular events and other clinical events during the entire follow-up period. If the patients could not respond to the questionnaire during regular office 
visits, the questionnaire was completed through personal telephone interviews. Other clinical information was obtained by reviewing hospital discharge reports relating to any other re-admission during the follow-up period. The performance of PCI or coronary artery bypass grafting (CABG) was validated by reviewing the original procedure protocols. Outcomes were adjudicated by two independent observers who were blinded with respect to the patients' baseline clinical and laboratory data.

\section{Statistical analysis}

Data normality was analyzed using the Kolmogorov-Smirnov test. Continuous normally distributed variables are described as mean \pm standard deviation, and non-normally distributed variables are described as median (interquartile range $[I Q R])$. Statistical differences in variables were compared using one-way ANOVA for normally distributed variables followed by Tukey's pairwise comparison. Categorical variables are presented as frequencies and percentages, and inter-group comparisons were tested using the chi-square test. Logarithmically transformed values of plasma FABP3, FABP4, visfatin, hs-CRP, adiponectin, and triglycerides were used in the statistical analysis since their distributions were skewed. Simple and multiple linear regression analyses were used to examine the associations and independence between plasma FABP3 level and values of the other parameters. Statistical significance was accepted if $p<0.05$. All data were analyzed using JMP version 10.0 for Windows (SAS Institute, Cary, NC, USA).

\section{Results}

Table 1 shows the clinical and demographic characteristics of the 249 patients with stable angina (males, 74.3\%; females, 25.7\%). The mean FABP3 level was $3.5 \mathrm{ng} / \mathrm{mL}$, and the median plasma FABP3 level was $1.3 \mathrm{ng} / \mathrm{mL}$ (IQR, $0.9 \mathrm{ng} / \mathrm{mL}$ to $3.1 \mathrm{ng} / \mathrm{mL}$ ). The prevalence rates of hypertension, hyperlipidemia, diabetes mellitus, left ventricular systolic dysfunction (LVSD), and abnormal QTc interval were 79.5\%, $66.7 \%, 47.8 \%, 16.3 \%$, and $31.7 \%$, respectively. The mean QTc interval was $442 \pm 37 \mathrm{~ms}$, and the mean EF was $61.8 \pm 12.6 \%$. The mean follow-up period was 41.3 months (range 5 to 96 months). During the follow-up period, 109 major adverse cardiovascular events occurred in the 249 stable angina patients, of which 24 $(9.6 \%)$ were death from all causes.

\section{Association between FABP3 and other parameters}

Simple linear regression analysis was performed to examine relationships between FABP3 and other variables (Table 2). The results showed that FABP3 was associated with age $(\beta=0.303, p<0.0001)$, the presence of diabetes mellitus $(\beta=0.330, p<0.0001)$, serum total cholesterol $(\beta=-0.176, p=0.006)$, HDL-C $(\beta=-0.172, p=0.007)$, LDL-C $(\beta=-0.193, p=0.002)$, albumin $(\beta=-0.382, p<0.0001)$, eGFR $(\beta=-0.653, p<$ $0.0001)$, hs-CRP $(\beta=0.303, p<0.0001)$, WBC count $(\beta$ $=0.407, p<0.0001)$, visfatin $(\beta=0.274, p=0.030)$, adiponectin $(\beta=0.419, p<0.0001)$, FABP4 $(\beta=0.769, p$ $<0.0001)$, heart rate $(\beta=0.359, p<0.0001)$, PR interval $(\beta=0.175, p=0.007)$, QTc interval $(\beta=0.359, p<$ 0.0001 , Figure 1A), left atrial diameter (LAD) $(\beta=$ $0.176, p=0.017)$, left ventricular mass index (LVMI) $(\beta$ $=0.205, p=0.012)$, interventricular septum thickness at end-diastole (IVSd) $(\beta=0.138, p=0.048)$, left ventricular posterior wall thickness at end-diastole (LVPWd) $(\beta=0.143, p=0.041)$, end-systolic volume $(\beta$ $=0.202, p=0.010)$, end-systolic volume index $(\beta=$ $0.234, p=0.003)$, left ventricular internal dimension at end-systole $(\beta=0.179, p=0.016)$, fractional shortening $(\beta=-0.222, p=0.001)$, and EF $(\beta=-0.225, p=0.002$, Figure 1B).

Table 1. Clinical and demographic characteristics of the study subjects

\begin{tabular}{|c|c|}
\hline Characteristic & Total $(\mathrm{N}=249)$ \\
\hline Sex (male/female) & $185 / 64$ \\
\hline Age (years) & $71.3 \pm 11.3$ \\
\hline Body mass index $\left(\mathrm{kg} / \mathrm{m}^{2}\right)$ & $25.7 \pm 3.9$ \\
\hline Waist (cm) & $92.1 \pm 10.1$ \\
\hline Hypertension (n, \%) & $198(79.5)$ \\
\hline Hyperlipidemia (n, \%) & $166(66.7)$ \\
\hline Diabetes mellitus (n, \%) & $119(47.8)$ \\
\hline Left ventricular systolic dysfunction (n, \%) & $30(16.3)$ \\
\hline Ejection fraction (\%) & $61.8 \pm 12.6$ \\
\hline Abnormal QTc interval (n, \%) & $79(31.7)$ \\
\hline Major adverse cardiovascular events, $\mathrm{n}(\%)$ & $109(43.8)$ \\
\hline All-cause mortality (n, \%) & $24(9.6)$ \\
\hline Currently smoking (n, \%) & $98(39.5)$ \\
\hline Corrected QT interval (ms) & $442 \pm 37$ \\
\hline Systolic blood pressure (mmHg) & $134 \pm 22$ \\
\hline Diastolic blood pressure (mmHg) & $76 \pm 12$ \\
\hline Fasting glucose (mg/dl) & $138.8 \pm 63.2$ \\
\hline HbA1c $(\%)$ & $6.9 \pm 1.5$ \\
\hline T-cholesterol (mg/dl) & $170.8 \pm 41.1$ \\
\hline Triglycerides (mg/dl) & $116.0(86.0-172.0)$ \\
\hline High-density lipoprotein-cholesterol (mg/dl) & $39.7 \pm 13.3$ \\
\hline Low-density lipoprotein -cholesterol (mg/dl) & $102.1 \pm 36.1$ \\
\hline Uric acid (mg/dl) & $6.7 \pm 2.2$ \\
\hline Creatinine (mg/dl) & $1.3(1.1-1.6)$ \\
\hline Estimated glomerular filtration rate $\left(\mathrm{ml} / \mathrm{min} / 1.73 \mathrm{~m}^{2}\right)$ & $55.5 \pm 20.3$ \\
\hline Albumin (g/dl) & $3.9 \pm 0.4$ \\
\hline Hematocrit (\%) & $39.1 \pm 5.4$ \\
\hline Hemoglobin (g/dl) & $13.1 \pm 2.0$ \\
\hline Fatty acid-binding protein $3(\mathrm{ng} / \mathrm{mL})$ & $1.3(0.9-3.1)$ \\
\hline Fatty acid-binding protein $4(\mathrm{ng} / \mathrm{mL})$ & $12.5(6.4-21.8)$ \\
\hline High-sensitivity C-reactive protein (mg/L) & $2.3(1.0-8.5)$ \\
\hline Adiponectin $(\mu \mathrm{g} / \mathrm{mL})$ & $4.6(2.0-10.1)$ \\
\hline White blood cell count $\left(\times 10^{9} / \mathrm{L}\right)$ & $7.291 \pm 2.500$ \\
\hline Anti-arrhythmic medication (n, \%) & $20(8.0)$ \\
\hline Beta-blockers (n, \%) & $99(39.8)$ \\
\hline
\end{tabular}




\begin{tabular}{ll}
\hline Characteristic & Total $(\mathrm{N}=249)$ \\
\hline Diuretics $(\mathrm{n}, \%)$ & $66(26.5)$ \\
Statins $(\mathrm{n}, \%)$ & $121(48.6)$ \\
\hline Data $\%$ expess
\end{tabular}

Data are expressed as mean $\pm \mathrm{SD}$, number (percentage), or median (interquartile range) for variables with a non-normal distribution.

Table 2. Simple linear regression analysis between fatty acidbinding protein 3 and other parameters

\begin{tabular}{|c|c|c|}
\hline \multirow[t]{2}{*}{ Parameter } & \multicolumn{2}{|c|}{$\begin{array}{l}\text { Fatty acid-binding } \\
\text { protein } 3\end{array}$} \\
\hline & $\beta$ & $p$-value \\
\hline Age & 0.303 & $<0.0001$ \\
\hline Sex & -0.083 & 0.194 \\
\hline Body mass index & 0.009 & 0.884 \\
\hline Diabetes Mellitus & 0.330 & $<0.0001$ \\
\hline Hypertension & 0.115 & 0.070 \\
\hline Hyperlipidemia & -0.104 & 0.101 \\
\hline Systolic blood pressure & 0.070 & 0.271 \\
\hline Diastolic blood pressure & -0.029 & 0.648 \\
\hline Total cholesterol & -0.176 & 0.006 \\
\hline High-density lipoprotein cholesterol & -0.172 & 0.007 \\
\hline Low-density lipoprotein cholesterol & -0.193 & 0.002 \\
\hline Triglycerides & -0.033 & 0.601 \\
\hline $\mathrm{HbA} 1 \mathrm{c}$ & 0.125 & 0.065 \\
\hline Albumin & -0.382 & $<0.0001$ \\
\hline Estimated GFR & -0.653 & $<0.0001$ \\
\hline High sensitivity C-reactive protein & 0.303 & $<0.0001$ \\
\hline White blood cell count & 0.407 & $<0.0001$ \\
\hline Visfatin & 0.274 & 0.030 \\
\hline Adiponectin & 0.419 & $<0.0001$ \\
\hline Fatty acid-binding protein 4 & 0.769 & $<0.0001$ \\
\hline \multicolumn{3}{|l|}{ ECG parameters } \\
\hline Heart Rate & 0.359 & $<0.0001$ \\
\hline PR interval & 0.175 & 0.007 \\
\hline QRS duration & 0.119 & 0.061 \\
\hline QT interval & 0.003 & 0.964 \\
\hline QTc interval & 0.359 & $<0.0001$ \\
\hline \multicolumn{3}{|l|}{ Echocardiographic parameters } \\
\hline Aortic root diameter & 0.100 & 0.213 \\
\hline Left atrial diameter & 0.176 & 0.017 \\
\hline Left ventricular mass index & 0.205 & 0.012 \\
\hline Interventricular septum thickness at end-diastole & 0.138 & 0.048 \\
\hline Left ventricular internal dimension at end-diastole & 0.123 & 0.136 \\
\hline Left ventricular posterior wall thickness at end-diastole & 0.143 & 0.041 \\
\hline End-diastolic volume & 0.110 & 0.194 \\
\hline End-diastolic volume index & 0.121 & 0.151 \\
\hline End-systolic volume & 0.202 & 0.010 \\
\hline End-systolic volume index & 0.234 & 0.003 \\
\hline Left ventricular internal dimension at end-systole & 0.179 & 0.016 \\
\hline Stroke volume & -0.002 & 0.982 \\
\hline Fractional shortening & -0.222 & 0.001 \\
\hline Ejection fraction & -0.225 & 0.002 \\
\hline Interventricular septum/Left ventricular posterior wall & -0.129 & 0.428 \\
\hline Ratio of $\mathrm{E}$ to $\mathrm{A}$ & 0.031 & 0.715 \\
\hline Peak E-wave velocity & -0.166 & 0.333 \\
\hline Peak A-wave velocity & 0.030 & 0.856 \\
\hline Peak velocity & 0.056 & 0.496 \\
\hline $\begin{array}{l}\text { Ratio of the left atrial dimension to the aortic annulus } \\
\text { dimension }\end{array}$ & 0.041 & 0.556 \\
\hline
\end{tabular}

Multiple linear regression analysis adjusted for age, sex, body mass index, diabetes mellitus, and hypertension revealed that FABP3 was positively associated with hs-CRP $(\beta=0.246, p<0.0001)$, WBC count $(\beta=0.394, p<0.0001)$, visfatin $(\beta=0.213, p=$ $0.044)$, adiponectin $(\beta=0.360, p<0.0001)$, FABP4 $(\beta=$ $0.705, p<0.0001)$, heart rate $(\beta=0.283, p<0.0001)$, QTc interval $(\beta=0.256, p<0.0001)$, LAD $(\beta=0.183, p$ $=0.011)$, LVMI $(\beta=0.151, p=0.048)$, end-systolic volume $(\beta=0.161, p=0.024)$, and end-systolic volume index $(\beta=0.168, p=0.022)$. In comparison, HDL-C $(\beta$ $=-0.121, p=0.049)$, fractional shortening $(\beta=-0.157, p$ $=0.017)$, and $\mathrm{EF}(\beta=-0.159, p=0.018)$ were negatively associated with FABP3 (Table 3).
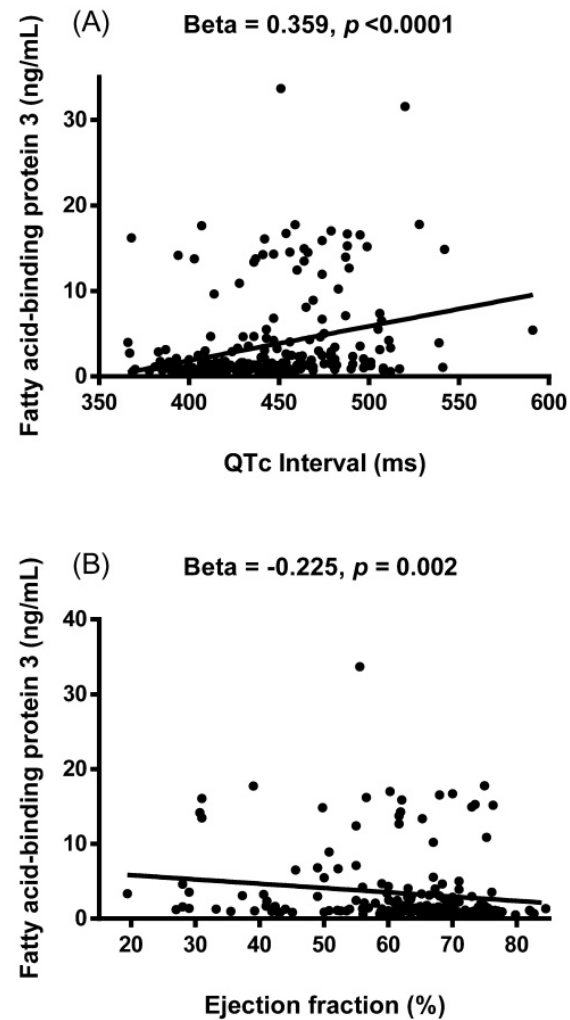

Figure 1. Associations between the concentration of plasma fatty acid-binding protein 3 (FABP3) and corrected QT (QTC) interval (A) and ejection fraction (B). The FABP3 concentration was positively associated with QTc interval and negatively associated with ejection fraction.

Table 3. Multiple linear regression analysis for fatty acid-binding protein 3, serum biomarkers, ECG parameters, and echocardiographic parameters

\begin{tabular}{lll}
\hline Parameter & \multicolumn{2}{l}{$\begin{array}{l}\text { Fatty acid-binding } \\
\text { protein } 3\end{array}$} \\
\cline { 2 - 3 } & $\beta^{*}$ & $p$-value \\
\hline Serum biomarkers & & \\
Total cholesterol & -0.068 & 0.268 \\
High-density lipoprotein cholesterol & -0.121 & 0.049 \\
Low-density lipoprotein cholesterol & -0.067 & 0.284 \\
Triglycerides & -0.028 & 0.643 \\
High sensitivity C-reactive protein & 0.246 & $<0.0001$ \\
White blood cell count & 0.394 & $<0.0001$ \\
Visfatin & 0.213 & 0.044 \\
Adiponectin & 0.360 & $<0.0001$ \\
Fatty acid-binding protein 4 & 0.705 & $<0.0001$ \\
ECG parameters & & \\
Heart Rate & 0.283 & $<0.0001$ \\
PR interval & 0.079 & 0.209 \\
QRS duration & 0.083 & 0.158 \\
\hline
\end{tabular}




\begin{tabular}{lll}
\hline Parameter & \multicolumn{2}{l}{$\begin{array}{l}\text { Fatty acid-binding } \\
\text { protein } 3\end{array}$} \\
\cline { 2 - 3 } & $\beta^{*}$ & $p$-value* \\
\hline QT interval & -0.021 & 0.719 \\
Corrected QT interval & 0.256 & $<0.0001$ \\
Echocardiographic parameters & & \\
Aortic root diameter & 0.066 & 0.419 \\
Left atrial diameter & 0.183 & 0.011 \\
Left ventricular mass index & 0.151 & 0.048 \\
Interventricular septum thickness at end-diastole & 0.082 & 0.236 \\
Left ventricular internal dimension at end-diastole & 0.114 & 0.149 \\
Left ventricular posterior wall thickness at end-diastole & 0.094 & 0.175 \\
End-diastolic volume & 0.105 & 0.193 \\
End-diastolic volume index & 0.093 & 0.244 \\
End-systolic volume & 0.161 & 0.024 \\
End-systolic volume index & 0.168 & 0.022 \\
Left ventricular internal dimension at end-systole & 0.134 & 0.051 \\
Stroke volume & 0.041 & 0.574 \\
Fractional shortening & -0.157 & 0.017 \\
Ejection fraction & -0.159 & 0.018 \\
Interventricular septum/Left ventricular posterior wall & -0.180 & 0.213 \\
Ratio of E to A & 0.055 & 0.502 \\
Peak E-wave velocity & -0.035 & 0.806 \\
Peak A-wave velocity & -0.105 & 0.437 \\
Peak velocity & 0.012 & 0.886 \\
Ratio of the left atrial dimension to the aortic annulus & 0.069 & 0.296 \\
dimension & & \\
\hline
\end{tabular}

*Adjusted for age, sex, body mass index, diabetes mellitus and hypertension by multiple linear regression analysis.

\section{Characteristics of the subjects according to the QTc prolongation status}

One hundred and nineteen patients were categorized as having a normal QTc interval, compared to 51 patients with borderline QTC prolongation and 79 patients with an abnormal QTC interval (Table 4). The abnormal QTc interval group had a significantly higher plasma FABP3 level than the borderline and normal QTc interval groups (2.6 $\mathrm{ng} / \mathrm{mL}$ [IQR 1.3 to 10.2 ] vs. $1.2 \mathrm{ng} / \mathrm{mL}$ [IQR 0.9 to 3.6] vs. $1.0 \mathrm{ng} / \mathrm{mL}$ [IQR 0.8 to 1.6], $p<0.0001$ ). In addition, the patients with an abnormal QTc interval were older and had higher rates of diabetes mellitus and LVSD. Moreover, the patients with an abnormal QTc interval (prolonged QT interval) had higher levels of blood urea nitrogen, creatinine, FABP4, hs-CRP, adiponectin, and WBC count than those with a normal QTc interval. In addition, the abnormal QTc interval group had a higher fasting glucose level than the borderline and normal QTc interval groups. The patients with an abnormal QTc interval had lower levels of LDL-cholesterol, albumin, hematocrit, hemoglobin, and eGFR than those with a normal QTc interval. Furthermore, the patients with an abnormal QTc interval also had lower levels of sodium than those with a borderline QTc interval. There were no significant differences in sex, hypertension, hyperlipidemia, current smoking, BMI, waist circumference, systolic blood pressure, diastolic blood pressure, levels of potassium, calcium, $\mathrm{HbA1c}$, total cholesterol, triglycerides, HDL-C, uric acid,

troponin-I, number of diseased coronary arteries, Gensini score, number of stents, and receiving anti-arrhythmic medication, beta-blockers, diuretics, and statins among the three groups. Furthermore, all of the participants had stable angina with a good thrombolysis in myocardial infarction (TIMI) flow grade. As a result, there was no significant deterioration or difference between the TIMI flow grade before and after coronary angiography or angioplasty. Moreover, because all of our electrocardiographic parameters were collected before the examination, we believe that changes in TIMI flow grade would not affect our results and conclusions. In addition, when we divided the patients into groups with or without LVSD, those with LVSD had a higher level of FABP3 than those without LVSD $(2.0 \mathrm{ng} / \mathrm{mL}$ [IQR 1.2 to 5.8 ] vs. $1.3 \mathrm{ng} / \mathrm{mL}$ [IQR 0.9 to 2.9 ], $p=$ 0.027) (data not shown).

Table 4. Baseline characteristics of the study population stratified by category of QTc prolongation status at baseline*

\begin{tabular}{|c|c|c|c|c|}
\hline Variable & Normal & Borderline & Abnormal & $P$-value \\
\hline No & 119 & 51 & 79 & \\
\hline Sex (male/female) & $88 / 31$ & $42 / 9$ & $55 / 24$ & 0.266 \\
\hline Age (years) & $68.4 \pm 11.3$ & $73.7 \pm 11.0$ & $74.1 \pm 10.6$ & 0.001 \\
\hline Age range & $42-99$ & $51-93$ & $40-94$ & \\
\hline $\begin{array}{l}\text { Body mass index } \\
\left(\mathrm{kg} / \mathrm{m}^{2}\right)\end{array}$ & $25.7 \pm 3.7$ & $25.6 \pm 3.9$ & $25.7 \pm 4.3$ & 0.988 \\
\hline Waist (cm) & $91.6 \pm 9.9$ & $93.0 \pm 8.9$ & $92.2 \pm 11.3$ & 0.760 \\
\hline $\begin{array}{l}\text { Hypertension } \\
(\mathrm{n}, \%)\end{array}$ & $91(76.5)$ & $42(82.4)$ & $65(82.3)$ & 0.522 \\
\hline $\begin{array}{l}\text { Hyperlipidemia } \\
(\mathrm{n}, \%)\end{array}$ & $85(71.4)$ & $32(62.8)$ & $49(62.0)$ & 0.311 \\
\hline $\begin{array}{l}\text { Diabetes mellitus } \\
(n, \%)\end{array}$ & $45(37.8)$ & $24(47.1)$ & $50(63.3)$ & 0.002 \\
\hline LVSD (n, \%) & $10(8.4)$ & $9(17.7)$ & $22(27.9)$ & 0.013 \\
\hline $\begin{array}{l}\text { Current smoking } \\
(\mathrm{n}, \%)\end{array}$ & $46(38.7)$ & $22(43.1)$ & $30(38.0)$ & 0.765 \\
\hline $\begin{array}{l}\text { Corrected QT } \\
\text { interval (ms) }\end{array}$ & $413 \pm 19$ & $445 \pm 9$ & $484 \pm 26$ & $<0.0001$ \\
\hline $\begin{array}{l}\text { Systolic blood } \\
\text { pressure }(\mathrm{mmHg})\end{array}$ & $134 \pm 19$ & $133 \pm 25$ & $135 \pm 24$ & 0.836 \\
\hline $\begin{array}{l}\text { Diastolic blood } \\
\text { pressure }(\mathrm{mmHg})\end{array}$ & $76 \pm 12$ & $76 \pm 10$ & $75 \pm 13$ & 0.884 \\
\hline Sodium (mEq/L) & $139.1 \pm 3.7$ & $140.2 \pm 4.5$ & $138.3 \pm 3.9$ & 0.026 \\
\hline $\begin{array}{l}\text { Potassium } \\
(\mathrm{mEq} / \mathrm{L})\end{array}$ & $3.8 \pm 0.4$ & $3.8 \pm 0.5$ & $3.9 \pm 1.0$ & 0.661 \\
\hline Calcium (mg/dl) & $8.7 \pm 0.9$ & $8.6 \pm 1.1$ & $8.6 \pm 0.9$ & 0.736 \\
\hline $\begin{array}{l}\text { Fasting glucose } \\
(\mathrm{mg} / \mathrm{dl})\end{array}$ & $129.4 \pm 54.0$ & $127.7 \pm 40.6$ & $160.2 \pm 80.9$ & 0.001 \\
\hline HbA1c (\%) & $6.8 \pm 1.5$ & $6.8 \pm 1.2$ & $7.2 \pm 1.6$ & 0.280 \\
\hline $\begin{array}{l}\text { T-cholesterol } \\
(\mathrm{mg} / \mathrm{dl})\end{array}$ & $175.6 \pm 43.5$ & $168.9 \pm 34.9$ & $164.8 \pm 40.7$ & 0.183 \\
\hline $\begin{array}{l}\text { Triglyceride } \\
(\mathrm{mg} / \mathrm{dl})\end{array}$ & $117.0(84.0-175.0)$ & $115.0(86.0-179.0)$ & $115.0(86.0-164.0)$ & 0.664 \\
\hline $\begin{array}{l}\text { HDL-cholesterol } \\
(\mathrm{mg} / \mathrm{dl})\end{array}$ & $40.7 \pm 15.3$ & $39.9 \pm 10.5$ & $38.0 \pm 11.6$ & 0.375 \\
\hline $\begin{array}{l}\text { LDL-cholesterol } \\
(\mathrm{mg} / \mathrm{dl})\end{array}$ & $108.0 \pm 40.8$ & $99.7 \pm 27.6$ & $94.6 \pm 31.8$ & 0.036 \\
\hline Uric acid (mg/dl) & $6.4 \pm 1.5$ & $6.9 \pm 3.3$ & $7.1 \pm 2.0$ & 0.098 \\
\hline $\begin{array}{l}\text { Blood urea } \\
\text { nitrogen }(\mathrm{mg} / \mathrm{dl})\end{array}$ & $19.3 \pm 10.6$ & $23.4 \pm 10.9$ & $26.7 \pm 16.5$ & 0.001 \\
\hline $\begin{array}{l}\text { Creatinine } \\
(\mathrm{mg} / \mathrm{dl})\end{array}$ & $1.2(1.1-1.4)$ & $1.3(1.1-1.5)$ & $1.4(1.2-1.8)$ & 0.003 \\
\hline Albumin (g/dl) & $4.1 \pm 0.4$ & $3.9 \pm 0.5$ & $3.8 \pm 0.4$ & 0.0002 \\
\hline Hematocrit (\%) & $40.1 \pm 4.3$ & $39.5 \pm 5.0$ & $37.4 \pm 6.6$ & 0.002 \\
\hline $\begin{array}{l}\text { Hemoglobin } \\
(\mathrm{g} / \mathrm{dl})\end{array}$ & $13.4 \pm 1.7$ & $13.2 \pm 1.9$ & $12.4 \pm 2.4$ & 0.002 \\
\hline
\end{tabular}




\begin{tabular}{|c|c|c|c|c|}
\hline Variable & Normal & Borderline & Abnormal & $P$-value \\
\hline $\begin{array}{l}\text { Estimated GFR } \\
\left(\mathrm{ml} / \mathrm{min} / 1.73 \mathrm{~m}^{2}\right)\end{array}$ & $61.0 \pm 17.5$ & $55.0 \pm 18.6$ & $47.4 \pm 22.5$ & $<0.0001$ \\
\hline $\begin{array}{l}\text { Fatty acid-binding } \\
\text { protein } 3(\mathrm{ng} / \mathrm{mL})\end{array}$ & $1.0(0.8-1.6)$ & $1.2(0.9-3.6)$ & $2.6(1.3-10.2)$ & $<0.0001$ \\
\hline $\begin{array}{l}\text { Fatty acid-binding } \\
\text { protein } 4(\mathrm{ng} / \mathrm{mL})\end{array}$ & $9.1(5.0-13.6)$ & $15.9(9.3-27.0)$ & $17.8(9.9-49.7)$ & 0.003 \\
\hline $\begin{array}{l}\text { Troponin-I } \\
\text { (ng/mL) }\end{array}$ & $0.1(0.0-0.4)$ & $0.1(0.0-0.4)$ & $0.1(0.0-0.3)$ & 0.376 \\
\hline Hs-CRP (mg/L) & $1.5(0.8-4.4)$ & $2.2(0.8-7.4)$ & $4.4(1.1-10.2)$ & 0.012 \\
\hline $\begin{array}{l}\text { Adiponectin } \\
(\mu \mathrm{g} / \mathrm{mL})\end{array}$ & $3.7(1.9-7.4)$ & $4.0(1.6-8.8)$ & $7.4(3.1-14.7)$ & 0.007 \\
\hline $\begin{array}{l}\text { White blood cell } \\
\text { count }\left(\times 10^{9} / \mathrm{L}\right)\end{array}$ & $6.907 \pm 1.895$ & $7.032 \pm 2.354$ & $8.037 \pm 3.171$ & 0.005 \\
\hline $\begin{array}{l}\text { No. of diseased } \\
\text { coronary arteries }\end{array}$ & $1.9 \pm 1.1$ & $2.3 \pm 0.9$ & $2.2 \pm 0.9$ & 0.061 \\
\hline Gensini score & $30.0(14.6-65.1)$ & $51.0(17.0-86.0)$ & $41.5(18.0-83.0)$ & 0.713 \\
\hline Number of stent & $0(0-1)$ & $0(0-1)$ & $0(0-0.0)$ & 0.065 \\
\hline $\begin{array}{l}\text { Anti-arrhythmic } \\
\text { medication }(n, \%)\end{array}$ & $7(5.9)$ & $4(7.8)$ & $9(11.4)$ & 0.376 \\
\hline $\begin{array}{l}\text { Beta-blockers } \\
(n, \%)\end{array}$ & $54(45.4)$ & $21(41.2)$ & $24(30.4)$ & 0.105 \\
\hline Diuretics (n, \%) & $24(20.2)$ & $16(31.4)$ & $26(32.9)$ & 0.094 \\
\hline Statins (n, \%) & $65(54.6)$ & $26(51.0)$ & $30(38.0)$ & 0.067 \\
\hline
\end{tabular}

Data are expressed as mean $\pm \mathrm{SD}$, number (percentage), or median (interquartile range). LVSD, left ventricular systolic dysfunction; HDL, high-density lipoprotein LDL, low-density lipoprotein; GFR, glomerular filtration rate; Hs-CRP, high-sensitivity C-reactive protein. *Classification of QTc prolongation: normal men $\leq 430 \mathrm{~ms}$; women $\leq 450 \mathrm{~ms}$; borderline men $431-450 \mathrm{~ms}$; women $451-470 \mathrm{~ms}$; abnormal men $\geq 451 \mathrm{~ms}$; women $\geq 471 \mathrm{~ms}$.

\section{Demographic and clinical characteristics according to tertiles of FABP3}

Table 5 shows the patients classified according to tertiles of FABP3 as follows: low FABP3 $(\leq 1 \mathrm{ng} / \mathrm{mL})$, $\mathrm{n}=81$; medium FABP3 $(1.1 \mathrm{ng} / \mathrm{mL}$ to $2.1 \mathrm{ng} / \mathrm{mL}), \mathrm{n}=$ 85; and high FABP3 (>2.1 ng/mL), $\mathrm{n}=83$. The high FABP3 group had a significantly higher QTc interval than the medium and low FABP3 groups $(458.5 \pm 41.1$ ms vs. $439.4 \pm 31.7 \mathrm{~ms}$ vs. $427.4 \pm 31.1 \mathrm{~ms}, p<0.0001)$, and a significantly lower EF than the low FABP3 group $(58.3 \pm 13.6 \%$ vs. $66.1 \pm 10.1 \%, p=0.002)$. Furthermore, the high FABP3 group were older $(p<$ $0.0001)$ and had higher prevalence rates of hypertension $(p=0.007)$, diabetes mellitus $(p<0.0001)$, abnormal QTc interval $(p<0.0001), \operatorname{LVSD}(p=0.043)$, and all-cause mortality $(p<0.0001)$. Moreover, the high FABP3 group had higher levels of visfatin, PR interval, LAD, LVMI, IVSd, LVPWd, end-systolic volume, end-systolic volume index, and left ventricular internal dimension at end-systole (LVID), and lower levels of total cholesterol and fractional shortening than the low FABP3 group. In addition, the high FABP3 group had higher fasting glucose, hs-CRP, WBC count, adiponectin, FABP4, and heart rate, and lower levels of LDL-C, albumin, and eGFR than the medium and low FABP3 groups.

\section{Discussion}

In the current study, we found that plasma FABP3 levels were positively related to QTc interval in patients with stable angina and significantly increased in the patients with an abnormal QTc interval compared to those with borderline and normal QTc intervals. Furthermore, FABP3 levels were negatively related to $\mathrm{EF}$, and the patients with LVSD had higher levels of FABP3 than those without LVSD. Moreover, with increasing FABP3 levels, the patients had higher rates of hypertension, diabetes mellitus, abnormal QTc interval, LVSD, and all-cause mortality, and higher levels of hs-CRP, WBC count, and visfatin. These findings are consistent with current evidence that FABP3 can be considered to be a prognostic marker for arrhythmia, heart failure, AMI, and pulmonary embolism, all of which are associated with poor clinical outcomes [31,32-34], and that inflammation induced by FABP3 may lead to CVD [15].

Table 5. Demographic and clinical characteristics according to tertiles of fatty acid-binding protein 3

\begin{tabular}{|c|c|c|c|c|}
\hline Parameter & $\begin{array}{l}\text { Low FABP3 } \leq 1 \\
\mathrm{ng} / \mathrm{mL}\end{array}$ & $\begin{array}{l}\text { Medium FABP3 } \\
=1.1-2.1 \mathrm{ng} / \mathrm{mL}\end{array}$ & $\begin{array}{l}\text { High FABP3 }>2.1 \\
\mathrm{ng} / \mathrm{mL}\end{array}$ & $p$-value \\
\hline No. & 81 & 85 & 83 & \\
\hline Sex (male/female) & $66 / 15$ & $61 / 24$ & $58 / 25$ & 0.190 \\
\hline Age (years) & $63.7 \pm 9.7$ & $74.4 \pm 9.2$ & $75.4 \pm 11.2$ & $<0.0001$ \\
\hline $\begin{array}{l}\text { Hypertension } \\
(\mathrm{n}, \%)\end{array}$ & $55(67.9)$ & $72(84.7)$ & 71 (85.5) & 0.007 \\
\hline $\begin{array}{l}\text { Diabetes mellitus } \\
(\mathrm{n}, \%)\end{array}$ & $24(29.6)$ & $39(45.9)$ & $56(67.5)$ & $<0.0001$ \\
\hline $\begin{array}{l}\text { Hyperlipidemia } \\
(\mathrm{n}, \%)\end{array}$ & $55(67.9)$ & $62(72.9)$ & $49(59.0)$ & 0.154 \\
\hline $\begin{array}{l}\text { Abnormal QTc } \\
\text { interval (n, \%) }\end{array}$ & $12(14.8)$ & $23(27.1)$ & $44(53.0)$ & $<0.0001$ \\
\hline LVSD (n, \%) & $8(9.9)$ & $12(14.1)$ & $20(24.1)$ & 0.043 \\
\hline $\begin{array}{l}\text { Current smoker } \\
(\mathrm{n}, \%)\end{array}$ & $28(34.6)$ & $35(41.2)$ & $35(42.7)$ & 0.529 \\
\hline $\begin{array}{l}\text { No. of diseased } \\
\text { coronary arteries }\end{array}$ & $1.9 \pm 0.9$ & $2.1 \pm 1.0$ & $2.2 \pm 0.9$ & 0.053 \\
\hline Number of stents & $0(0-1)$ & $0(0-1)$ & $0(0-1)$ & 0.693 \\
\hline $\begin{array}{l}\text { Body mass index } \\
\left(\mathrm{kg} / \mathrm{m}^{2}\right)\end{array}$ & $25.2 \pm 3.4$ & $26.0 \pm 4.2$ & $25.9 \pm 4.2$ & 0.375 \\
\hline $\begin{array}{l}\text { Systolic BP } \\
(\mathrm{mmHg})\end{array}$ & $129 \pm 17$ & $138 \pm 20$ & $136 \pm 26$ & 0.019 \\
\hline $\begin{array}{l}\text { Diastolic BP } \\
(\mathrm{mmHg})\end{array}$ & $75 \pm 10$ & $77 \pm 10$ & $76 \pm 15$ & 0.779 \\
\hline $\begin{array}{l}\text { Total-cholesterol } \\
(\mathrm{mg} / \mathrm{dl})\end{array}$ & $179.7 \pm 45.1$ & $169.9 \pm 36.0$ & $163.0 \pm 40.8$ & 0.034 \\
\hline $\begin{array}{l}\text { Triglycerides } \\
\text { (mg/dl) }\end{array}$ & $119.0(84.5-180.0)$ & $112.0(86.0-162.5)$ & $115.0(85.5-172.0)$ & 0.867 \\
\hline $\begin{array}{l}\text { HDL-cholesterol } \\
(\mathrm{mg} / \mathrm{dl})\end{array}$ & $40.2 \pm 9.9$ & $41.1 \pm 16.2$ & $37.7 \pm 12.8$ & 0.238 \\
\hline $\begin{array}{l}\text { LDL-cholesterol } \\
(\mathrm{mg} / \mathrm{dl})\end{array}$ & $113.4 \pm 40.9$ & $99.0 \pm 32.1$ & $94.0 \pm 32.1$ & 0.002 \\
\hline $\begin{array}{l}\text { Fasting glucose } \\
(\mathrm{mg} / \mathrm{dl})\end{array}$ & $122.8 \pm 39.9$ & $131.9 \pm 58.8$ & $161.6 \pm 78.3$ & 0.0002 \\
\hline HbA1c (\%) & $6.6 \pm 1.2$ & $7.0 \pm 1.6$ & $7.2 \pm 1.5$ & 0.055 \\
\hline Albumin $(\mathrm{g} / \mathrm{dl})$ & $4.2 \pm 0.3$ & $4.0 \pm 0.4$ & $3.7 \pm 0.5$ & $<0.0001$ \\
\hline $\begin{array}{l}\text { Estimated GFR } \\
\left(\mathrm{ml} / \mathrm{min} / 1.73 \mathrm{~m}^{2}\right)\end{array}$ & $69.7 \pm 14.2$ & $57.3 \pm 14.5$ & $39.6 \pm 19.3$ & $<0.0001$ \\
\hline $\mathrm{Hs}-\mathrm{CRP}$ (mg/L) & $1.3(0.8-3.0)$ & $2.1(1.1-7.3)$ & $6.9(1.7-15.7)$ & 0.0001 \\
\hline $\begin{array}{l}\text { White blood cell } \\
\text { count }\left(\times 10^{9} / \mathrm{L}\right)\end{array}$ & $6.674 \pm 1.553$ & $6.679 \pm 1.856$ & $8.520 \pm 3.276$ & $<0.0001$ \\
\hline Visfatin $(\mathrm{ng} / \mathrm{mL})$ & $9.5(7.1-12.5)$ & $10.9(7.0-18.8)$ & $14.6(9.6-31.3)$ & 0.042 \\
\hline $\begin{array}{l}\text { Adiponectin } \\
(\mu \mathrm{g} / \mathrm{mL})\end{array}$ & $1.6(0.9-2.9)$ & $2.8(1.9-5.6)$ & $5.4(1.0-8.6)$ & $<0.0001$ \\
\hline FABP4 (ng/mL) & $5.4(4.4-8.9)$ & $12.8(8.4-16.1)$ & $42.5(18.3-66.1)$ & $<0.0001$ \\
\hline \multicolumn{5}{|l|}{ ECG parameters } \\
\hline Heart Rate (bpm) & $70.0 \pm 13.3$ & $70.9 \pm 14.0$ & $84.1 \pm 20.3$ & $<0.0001$ \\
\hline PR interval (ms) & $163.8 \pm 23.5$ & $171.8 \pm 31.1$ & $177.3 \pm 38.6$ & 0.029 \\
\hline QRS duration (ms) & $93.8 \pm 15.6$ & $96.4 \pm 19.1$ & $99.8 \pm 20.6$ & 0.114 \\
\hline QT interval (ms) & $392.6 \pm 60.5$ & $405.0 \pm 56.7$ & $395.3 \pm 51.0$ & 0.322 \\
\hline
\end{tabular}




\begin{tabular}{|c|c|c|c|c|}
\hline Parameter & $\begin{array}{l}\text { Low FABP3 } \leq 1 \\
\mathrm{ng} / \mathrm{mL}\end{array}$ & $\begin{array}{l}\text { Medium FABP3 } \\
=1.1-2.1 \mathrm{ng} / \mathrm{mL}\end{array}$ & $\begin{array}{l}\text { High FABP3 > } 2.1 \\
\mathrm{ng} / \mathrm{mL}\end{array}$ & $p$-value \\
\hline QTc interval (ms) & $427.4 \pm 31.1$ & $439.4 \pm 31.7$ & $458.5 \pm 41.1$ & $<0.0001$ \\
\hline \multicolumn{5}{|l|}{$\begin{array}{l}\text { Echocardiographic } \\
\text { parameters }\end{array}$} \\
\hline $\begin{array}{l}\text { Aortic root } \\
\text { diameter }(\mathrm{cm})\end{array}$ & $3.1 \pm 0.5$ & $3.3 \pm 0.5$ & $3.2 \pm 0.5$ & 0.088 \\
\hline $\begin{array}{l}\text { Left atrial diameter } \\
(\mathrm{cm})\end{array}$ & $3.7 \pm 0.6$ & $3.8 \pm 0.6$ & $4.0 \pm 0.7$ & 0.039 \\
\hline LVMI (g/m²) & $114.3 \pm 27.6$ & $128.1 \pm 42.6$ & $133.9 \pm 49.7$ & 0.047 \\
\hline IVSd (cm) & $1.1 \pm 0.2$ & $1.2 \pm 0.2$ & $1.2 \pm 0.2$ & 0.006 \\
\hline LVIDd (cm) & $4.8 \pm 0.5$ & $4.9 \pm 0.7$ & $5.0 \pm 0.8$ & 0.384 \\
\hline LVPWd (cm) & $1.0 \pm 0.2$ & $1.1 \pm 0.2$ & $1.1 \pm 0.2$ & 0.027 \\
\hline $\begin{array}{l}\text { End-diastolic } \\
\text { volume }(\mathrm{ml})\end{array}$ & $111.8 \pm 28.2$ & $115.7 \pm 44.0$ & $122.8 \pm 47.3$ & 0.421 \\
\hline $\begin{array}{l}\text { End-diastolic } \\
\text { volume index } \\
\left(\mathrm{ml} / \mathrm{m}^{2}\right)\end{array}$ & $64.7 \pm 17.1$ & $65.9 \pm 26.7$ & $70.6 \pm 28.2$ & 0.463 \\
\hline $\begin{array}{l}\text { End-systolic } \\
\text { volume (ml) }\end{array}$ & $39.9 \pm 19.5$ & $50.7 \pm 35.7$ & $57.1 \pm 38.6$ & 0.025 \\
\hline $\begin{array}{l}\text { End-systolic } \\
\text { volume index } \\
\left(\mathrm{ml} / \mathrm{m}^{2}\right)\end{array}$ & $23.2 \pm 12.9$ & $27.6 \pm 20.2$ & $32.7 \pm 21.5$ & 0.037 \\
\hline LVIDs (mm) & $3.1 \pm 0.6$ & $3.4 \pm 0.9$ & $3.5 \pm 1.0$ & 0.029 \\
\hline $\begin{array}{l}\text { Stroke volume } \\
(\mathrm{ml})\end{array}$ & $70.1 \pm 20.6$ & $67.3 \pm 20.2$ & $68.0 \pm 21.7$ & 0.764 \\
\hline $\begin{array}{l}\text { Fractional } \\
\text { shortening (\%) }\end{array}$ & $37.0 \pm 7.1$ & $33.5 \pm 8.8$ & $31.9 \pm 9.0$ & 0.002 \\
\hline $\begin{array}{l}\text { Ejection fraction } \\
(\%)\end{array}$ & $66.1 \pm 10.1$ & $61.3 \pm 12.9$ & $58.3 \pm 13.6$ & 0.002 \\
\hline IVS/LVPW & $1.1 \pm 0.2$ & $1.1 \pm 0.1$ & $1.1 \pm 0.1$ & 0.645 \\
\hline Ratio of E to A & $1.0 \pm 0.3$ & $1.0 \pm 0.6$ & $1.1 \pm 0.5$ & 0.474 \\
\hline $\begin{array}{l}\text { Peak E-wave } \\
\text { velocity }(\mathrm{cm} / \mathrm{s})\end{array}$ & $65.4 \pm 14.8$ & $67.1 \pm 20.7$ & $63.7 \pm 19.1$ & 0.921 \\
\hline $\begin{array}{l}\text { Peak A-wave } \\
\text { velocity }(\mathrm{cm} / \mathrm{s})\end{array}$ & $73.1 \pm 20.9$ & $82.8 \pm 23.7$ & $71.1 \pm 25.1$ & 0.413 \\
\hline $\begin{array}{l}\text { Peak velocity } \\
(\mathrm{cm} / \mathrm{s})\end{array}$ & $227.0 \pm 50.2$ & $226.7 \pm 43.9$ & $233.4 \pm 78.8$ & 0.817 \\
\hline $\mathrm{LA} / \mathrm{AO}$ & $1.2 \pm 0.2$ & $1.2 \pm 0.3$ & $1.2 \pm 0.3$ & 0.493 \\
\hline MACEs (n, \%) & $29(35.8)$ & 39 (45.9) & $41(49.4)$ & 0.191 \\
\hline $\begin{array}{l}\text { All-cause } \\
\text { mortality (n, \%) }\end{array}$ & $1(1.2)$ & $6(7.1)$ & $17(20.5)$ & $<0.0001$ \\
\hline
\end{tabular}

Data are expressed as mean $\pm \mathrm{SD}$, or number (percentage). LVSD, left ventricular systolic dysfunction; BP, blood pressure; HDL, high-density lipoprotein cholesterol; LDL, low-density lipoprotein cholesterol; GFR, glomerular filtration rate; Hs-CRP, high sensitivity C-reactive protein; FABP, fatty acid-binding protein; QTc, corrected QT; LVMI, left ventricular mass index; IVSd, interventricular septum thickness at end-diastole; LVIDd, left ventricular internal dimension at end-diastole, LVPWd, left ventricular posterior wall thickness at end-diastole; LVIDs, Left ventricular internal dimension at end-systole; IVS/LVPW, interventricular septum/Left ventricular posterior wall; LA/AO, ratio of the left atrial dimension to the aortic annulus dimension; MACEs, major adverse cardiovascular events. Abnormal QTc interval defined as men $\geq 451 \mathrm{~ms}$; women $\geq 471 \mathrm{~ms}$; Left ventricular systolic dysfunction defined as left ventricular ejection fraction $\leq 50 \%$.

Previous studies have shown that $\mathrm{AF}$ and premature ventricular contractions can induce FABP3 leakage [35,36], and ventricular cell loss caused by apoptosis has been confirmed in AF tachycardiainduced cardiomyopathy $[37,38]$. In addition, cardiac sympathetic nervous scintigraphy findings have shown that AF directly activates cardiac sympathetic nervous function in patients with isolated AF [39]. Hence, AF-induced apoptosis and sympathetic nervous system activation may contribute to FABP3 leakage in patients with AF. Furthermore, FABP3 has been reported to be elevated in patients with Brugada syndrome and ventricular fibrillation despite the absence of structural heart disease and cardiac dysfunction [32]. Therefore, assessing FABP3 could provide useful information in patients with arrhythmia. Moreover, previous studies have reported associations between proinflammatory cytokines (such as IL-6 and CRP) and arrhythmias through the modulation of ion channel function $[9,40]$ and the aggravation of sympathetic tone [40]. Myocardial fibrosis, which has been associated with inflammatory processes, can affect ventricular conduction causing a delay in repolarization that could lead to ventricular arrhythmias [40,41]. Our findings suggest that inflammatory activity as reflected by hs-CRP and WBC count was a strong etiological factor in the association between higher levels of plasma FABP3 and QTC prolongation. In addition, in our previous study, we found that plasma FABP4 levels were significantly higher in patients with an abnormal QTc interval, and that they were correlated with QTc prolongation [42]. In the present study, a higher FABP3 level was positively associated with plasma FABP4 level, thereby raising the possibility that FABP3 may play a role in QTc interval prolongation.

In the present study, FABP3 levels were negatively associated with EF and the patients with LVSD had higher levels of FABP3 than those without LVSD. These data are consistent with those of Arimoto et al. who reported higher serum levels of FABP3 in patients with heart failure, and that this was related to the severity of heart failure [31]. During the development of cardiac hypertrophy and failure, a transition of energy substrate utilization occurs with reduced fatty acid oxidation and increased glucose utilization $[43,44]$. Binas et al. reported that both cellular uptake and lipid oxidation of long-chain fatty acids were severely depressed in FABP3 knockout mice [45], indicating that FABP3 plays a critical role in the uptake and transport of long-chain fatty acids in cardiomyocytes. In addition, inflammation is known to play a central role in the development of heart failure [46]. In this study, a higher FABP3 level was independently associated with plasma levels of hs-CRP, visfatin, and WBC count, which suggests that FABP3 may act through inflammatory responses to play an important role in the pathophysiology of reduced $\mathrm{EF}$ in patients with stable angina.

In this study, we also found that a high FABP3 level was significantly associated with the occurrence of all-cause mortality in patients with stable angina and that a higher FABP3 level was positively associated with the plasma level of adiponectin. In our previous study, we showed that high adiponectin plasma concentrations were independently associated with major adverse cardiovascular events in patients with CAD and type 2 diabetes mellitus [47]. Suzuki et al. proposed that FABP3 could be used as a biomarker 
to independently predict adverse cardiac events within 30 days in patients with ACS [48]. In addition, O'Donoghue et al. found that elevated FABP3 was associated with increased risks of death and other adverse cardiac events among patients with ACS, and that FABP3 alone could be used to evaluate the risk [49]. Kilcullen et al. also found that FABP3 could be used to identify patients at high risk of adverse outcomes, and that proper attention should be paid to these patients to improve their prognosis [50]. Furthermore, Viswanathan et al. reported that when used in combination with troponin, FABP3 measurements could help with predicting both long-term mortality and the likelihood of re-infarction in the presence of suspected ACS, even for low- to intermediate-risk subjects [51]. Moreover, Agnello et al. suggested that FABP3 may be a good candidate to rule in or rule out AMI at the emergency department [52]. However, in contrast, a literature review showed that FABP3 is clearly not a reliable marker of ACS, since it cannot be used to diagnose AMI, either as a stand-alone test or combined with hs-cardiac troponin [53]. Further investigations are needed to clarify this issue.

The current study has some limitations. First, the number of enrolled patients was relatively small. Additional studies are necessary to prospectively examine whether a reduction in plasma FABP3 concentrations can decrease the rates of abnormal QTC interval and LVSD, and improve systolic function. Second, we enrolled individuals in whom coronary angiography was clinically indicated. This population is at an intermediate-high risk of future cardiovascular events, and our findings may not apply to lower risk individuals. Third, all of the patients' ECG parameters including QT interval and QTc were measured by the same medical technician using an identical computer-based method. As a result, we did not evaluate the variability in measured QT intervals. Fourth, whether FABP3 is associated with cardiomyocyte electrophysiology such as cardiac ion channels is still unclear, and further studies are needed to elucidate the underlying mechanisms. Furthermore, if the study population had different diseases (e.g. ACS and myocardial infarction), the diverse disease severity and condition of the study population may have impacted the results. To avoid selection bias, we chose individuals with stable angina for this study, thus the results of the present study might not be generalizable to other populations.

\section{Conclusions}

In conclusion, elevated plasma FABP3 was closely associated with prolonged QTc Interval and reduced $\mathrm{EF}$ in patients with stable angina, and was independently associated with plasma levels of hs-CRP, visfatin, FABP4, and WBC count, suggesting that plasma FABP3 may act through the effects of inflammation or cardiomyocyte injury to play as a surrogate parameter of prolonged QTc Interval and reduced EF. The mechanisms by which FABP3 level and QTc interval and EF are linked remain to be investigated.

\section{Supplementary Material}

Supplementary materials. http://www.medsci.org/v18p2076s1.pdf

\section{Acknowledgements}

The authors would like to thank E-Da Hospital of the Republic of China, Taiwan, for financially supporting this research under contracts EDAHI108001 and EDAHI109002. We appreciate the assistance of the staff and members of the heart care team for their help in various measurements and other organizational aspects of this study.

\section{Competing Interests}

The authors have declared that no competing interest exists.

\section{References}

1. Hojat M, Jahromi MK, Koshkaki SR, Rahmanian M. Comparison of risk factors of cardiovascular diseases in male and female nurses. J Educ Health Promot. 2019; 8: 19.

2. Suárez C. Baseline characteristics of patients with cerebrovascular disease in the REACH registry: The Spanish contribution. Cerebrovasc Dis. 2007; 24: 89-95.

3. Jahromi MK, Hojat M, Koshkaki SR, Nazari F, Ragibnejad M. Risk factors of heart disease in nurses. Iran J Nurs Midwifery Res. 2017; 22: 332-7.

4. Jessup M, Brozena S. Heart failure. N Engl J Med. 2003; 348: 2007-18.

5. Straburzyńska-Migaj E, Kałużna-Oleksy M, Maggioni AP, Grajek S, Opolski G, Ponikowski P, et al. Patients with heart failure and concomitant chronic obstructive pulmonary disease participating in the Heart Failure Pilot Survey (ESC-HF Pilot)-Polish population. Arch Med Sci. 2015; 11: 743-50.

6. Im SW, Lee MK, Lee HJ, Oh SI, Kim HL, Sung J, et al. Analysis of genetic and non-genetic factors that affect the QTc interval in a Mongolian population: the GENDISCAN study. Exp Mol Med. 2009; 41: 841-8.

7. Skrzynia C, Berg JS, Willis MS, Jensen BC. Genetics and heart failure: a concise guide for the clinician. Curr Cardiol Rev. 2015; 11: 10-7.

8. Hoffman BF, Feinmark SJ, Guo SD. Electrophysiologic effects of interactions between activated canine neutrophils and cardiac myocytes. J Cardiovasc Electrophysiol. 1997; 8: 679-87.

9. Hoffman BF, Guo SD, Feinmark SJ. Arrhythmias caused by platelet activating factor. J Cardiovasc Electrophysiol. 1996; 7: 120-33.

10. Chung MK, Martin DO, Sprecher D, Wazni O, Kanderian A, Carnes CA, et al. C-reactive protein elevation in patients with atrial arrhythmias: inflammatory mechanisms and persistence of atrial fibrillation. Circulation. 2001; 104: 2886-91

11. Arroyo-Espliguero R, Avanzas P, Quiles J, Kaski JC. C-reactive protein predicts functional status and correlates with left ventricular ejection fraction in patients with chronic stable angina. Atherosclerosis. 2009; 205: 319-24.

12. Tbahriti HF, Meknassi D, Moussaoui R, Messaoudi A, Zemour L, Kaddous A, et al. Inflammatory status in chronic renal failure: the role of homocysteinemia and pro-inflammatory cytokines. World J Nephrol. 2013; 2: 31-7.

13. Chmurzynska A. The multigene family of fatty acid-binding proteins (FABPs): function, structure and polymorphism. J Appl Genet. 2006; 47: 39-48.

14. Schernthaner C, Lichtenauer M, Wernly B, Paar V, Pistulli R, Rohm I, et al. Multibiomarker analysis in patients with acute myocardial infarction. Eur J Clin Invest. 2017; 47: 638-48.

15. Chen K, Chen QJ, Wang LJ, Liu ZH, Zhang Q, Yang K, et al. Increment of HFABP level in coronary artery in-stent restenosis segments in diabetic and nondiabetic minipigs: HFABP overexpression promotes multiple pathwayrelated inflammation, growth and migration in human vascular smooth muscle cells. J Vasc Res. 2016; 53: 27-38. 
16. Wang CP, Lu LF, Yu TH, Hung WC, Chiu CA, Chung FM, et al. Serum levels of total p-cresylsulphate are associated with angiographic coronary atherosclerosis severity in stable angina patients with early stage of renal failure. Atherosclerosis. 2010; 211: 579-83.

17. Gibbons RJ, Chatterjee K, Daley J, Douglas JS, Fihn SD, Gardin JM, et al. ACC/AHA/ACP-ASIM guidelines for the management of patients with chronic stable angina: executive summary and recommendations. A Report of the American College of Cardiology/American Heart Association Task Force on Practice Guidelines (Committee on Management of Patients with Chronic Stable Angina). Circulation. 1999; 99: 2829-48.

18. The Thrombolysis in Myocardial Infarction (TIMI) trial. Phase I findings. TIMI Study Group. N Engl J Med. 1985; 312: 932-6.

19. Thygesen K, Alpert JS, White HD; Joint ESC/ACCF/AHA/WHF Task Force for the Redefinition of Myocardial Infarction. Universal definition of myocardial infarction. J Am Coll Cardiol. 2007; 50: 2173-95.

20. Bonow RO, Carabello BA, Chatterjee K, de Leon AC Jr, Faxon DP, Freed MD, et al. American College of Cardiology/American Heart Association Task Force on Practice Guidelines. 2008 Focused update incorporated into the ACC/AHA2006 guidelines for the management of patients with valvular heart disease: a report of the American College of Cardiology/American Heart Association Task Force on Practice Guidelines (Writing Committee to revise the 1998 guidelines for the management of patients with valvular heart disease). Endorsed by the Society of Cardiovascular Anesthesiologists, Society for Cardiovascular Angiography and Interventions, and Society of Thoracic Surgeons. J Am Coll Cardiol. 2008; 52: e1-142.

21. American Diabetes Association. Diagnosis and classification of diabetes mellitus. Diabetes Care. 2012; 35 (Suppl 1): S64-S71.

22. Kong $\mathrm{X}, \mathrm{Ma} \mathrm{Y}, \mathrm{Chen} \mathrm{J}$, Luo $\mathrm{Q}, \mathrm{Yu}$ X, Li Y, et al. Chinese eGFR Investigation Collaboration. Evaluation of the chronic kidney disease epidemiology collaboration equation for estimating glomerular filtration rate in the Chinese population. Nephrol Dial Transplant. 2013; 28: 641-51.

23. Rautaharju PM, Surawicz B, Gettes LS, Bailey JJ, Childers R, Deal BJ, et al. $\mathrm{AHA} / \mathrm{ACCF} / \mathrm{HRS}$ recommendations for the standardization and interpretation of the electrocardiogram: part IV: the ST segment, $\mathrm{T}$ and U waves, and the QT interval: a scientific statement from the American Heart Association Electrocardiography and Arrhythmias Committee, Council on Clinical Cardiology; the American College of Cardiology Foundation; and the Heart Rhythm Society. Endorsed by the International Society for Computerized Electrocardiology. J Am Coll Cardiol. 2009; 53: 982-91.

24. Naksuk N, Hu T, Krittanawong C, Thongprayoon C, Sharma S, Park JY, et al. Association of Serum Magnesium on Mortality in Patients Admitted to the Intensive Cardiac Care Unit. Am J Med. 2017; 130: 229.e5-229.e13.

25. Salvi V, Karnad DR, Panicker GK, Natekar M, Hingorani P, Kerkar V, et al. Comparison of 5 methods of QT interval measurements on electrocardiograms from a thorough QT/QTc study: effect on assay sensitivity and categorical outliers. J Electrocardiol. 2011; 44: 96-104.

26. Malik M, Hnatkova K, Batchvarov V, Gang Y, Smetana P, Camm AJ. Sample size, power calculations, and their implications for the cost of thorough studies of drug induced QT interval prolongation. Pacing Clin Electrophysiol. 2004; 27: $1659-69$

27. Straus SM, Kors JA, De Bruin ML, van der Hooft CS, Hofman A, Heeringa J, et al. Prolonged QTc interval and risk of sudden cardiac death in a population of older adults. J Am Coll Cardiol. 2006; 47: 362-7.

28. Gardin JM, Adams DB, Douglas PS, Feigenbaum H, Forst DH, Fraser AG, et al. American Society of Echocardiography. Recommendations for a standardized report for adult transthoracic echocardiography: a report from the American Society of Echocardiography's Nomenclature and Standards Committee and Task Force for a Standardized Echocardiography Report. J Am Soc Echocardiogr. 2002; 15: 275-90.

29. Kasner M, Wassermann D, Steendijk P, Gaub R, Wilkenshoff U, Weitmann K, et al. Utility of Doppler echocardiography and tissue Doppler imaging in the estimation of diastolic function in heart failure with normal ejection fraction: a comparative Doppler-conductance catheterization study. Circulation. 2007; 116: 637-47.

30. Vasan RS, Levy D. Defining diastolic heart failure: a call for standardized diagnostic criteria. Circulation. 2000; 101: 2118-21.

31. Arimoto T, Takeishi Y, Shiga R, Fukui A, Tachibana H, Nozaki N, et al. Prognostic value of elevated circulating heart-type fatty acid binding protein in patients with congestive heart failure. J Card Fail. 2005; 11: 56-60.

32. Daidoji H, Arimoto T, Iwayama T, Ishigaki D, Hashimoto N, Kumagai Y, et al. Circulating heart-type fatty acid-binding protein levels predict ventricular fibrillation in Brugada syndrome. J Cardiol. 2016; 67: 221-8.

33. Ye XD, He Y, Wang S, Wong GT, Irwin MG, Xia Z. Heart-type fatty acid binding protein (H-FABP) as a biomarker for acute myocardial injury and long-term post-ischemic prognosis. Acta Pharmacol Sin. 2018; 39: 1155-63.

34. Qian HY, Huang J, Yang YJ, Yang YM, Li ZZ, Zhang JM. Heart-type Fatty Acid Binding Protein in the Assessment of Acute Pulmonary Embolism. Am J Med Sci. 2016; 352: 557-62.

35. Otaki $\mathrm{Y}$, Arimoto $\mathrm{T}$, Takahashi $\mathrm{H}$, Kadowaki $\mathrm{S}$, Ishigaki $\mathrm{D}$, Narumi $\mathrm{T}$, et al. Prognostic value of myocardial damage markers in patients with chronic heart failure with atrial fibrillation. Intern Med. 2014; 53: 661-8.

36. Niizeki $\mathrm{T}$, Takeishi $\mathrm{Y}$, Takabatake $\mathrm{N}$, Shibata $\mathrm{Y}$, Konta $\mathrm{T}$, Kato $\mathrm{T}$, et al. Circulating levels of heart-type fatty acid-binding protein in a general Japanese population: effects of age, gender, and physiologic characteristics. Circ J. 2007; 71: 1452-7.
37. Trappe K, Thomas D, Bikou O, Kelemen K, Lugenbiel P, Voss F, et al. Suppression of persistent atrial fibrillation by genetic knockdown of caspase 3: a pre-clinical pilot study. Eur Heart J. 2013; 34: 147-57.

38. Bauer A, McDonald AD, Donahue JK. Pathophysiological findings in a model of persistent atrial fibrillation and severe congestive heart failure. Cardiovasc Res. 2004; 61: 764-70.

39. Arimoto $\mathrm{T}$, Tada $\mathrm{H}$, Igarashi $\mathrm{M}$, Sekiguchi $\mathrm{Y}$, Sato A, Koyama $\mathrm{T}$, et al. High washout rate of iodine-123-metaiodobenzylguanidine imaging predicts the outcome of catheter ablation of atrial fibrillation. J Cardiovasc Electrophysiol. 2011; 22: 1297-304.

40. Parekh RS, Plantinga LC, Kao WH, Meoni LA, Jaar BG, Fink NE, et al. The association of sudden cardiac death with inflammation and other traditional risk factors. Kidney Int. 2008; 74: 1335-42.

41. NaghaviM, Libby P, Falk E, Casscells SW, Litovsky S, Rumberger J, et al. From vulnerable plaque to vulnerable patient: a call for new definitions and risk assessment strategies: Part II. Circulation. 2003; 108: 1772-8.

42. Wang CP, Hsu CC, Hung WC, Yu TH, Wu CC, Tsai IT, et al. Plasma fatty acidbinding protein 4 (FABP4) level is associated with abnormal QTc interval in patients with stable angina and chronic kidney disease. BMC Cardiovasc Disord. 2019; 19: 153.

43. Sack MN, Kelly DP. The energy substrate switch during development of heart failure: gene regulatory mechanisms. Int J Mol Med. 1998; 1: 17-24.

44. Sack MN, Rader TA, Park S, Bastin J, McCune SA, Kelly DP. Fatty acid oxidation enzyme gene expression is downregulated in the failing heart. Circulation. 1996; 94: 2837-42

45. Binas B, Danneberg H, McWhir J, Mullins L, Clark AJ. Requirement for the heart-type fatty acid binding protein in cardiac fatty acid utilization. FASEB J. 1999; 13: 805-12.

46. Riehle C, Bauersachs J. Key inflammatory mechanisms underlying heart failure. Herz. 2019; 44: 96-106

47. Hung WC, Wang CP, Lu LF, Yu TH, Chiu CA, Chung FM, et al. Circulating adiponectin level is associated with major adverse cardiovascular events in type 2 diabetic patients with coronary artery disease. Endocr J. 2010; 57: 793-802

48. Suzuki M, Hori S, Noma S, Kobayashi K. Prognostic value of a qualitative test for heart-type fatty acid-binding protein in patients with acute coronary syndrome. Int Heart J. 2005; 46: 601-6.

49. O'Donoghue M, de Lemos JA, Morrow DA, Murphy SA, Buros JL, Cannon CP, et al. Prognostic utility of heart-type fatty acid binding protein in patients with acute coronary syndromes. Circulation. 2006; 114: 550-7.

50. Kilcullen N, Viswanathan $\mathrm{K}$, Das $\mathrm{R}$, Morrell $\mathrm{C}$, Farrin A, Barth JH, et al. Heart-type fatty acid-binding protein predicts long-term mortality after acute coronary syndrome and identifies high-risk patients across the range of troponin values. J Am Coll Cardiol. 2007; 50: 2061-7.

51. Viswanathan $\mathrm{K}$, Kilcullen N, Morrell C, Thistlethwaite SJ, Sivananthan MU, Hassan TB, et al. Heart-type fatty acid-binding protein predicts long-term mortality and re-infarction in consecutive patients with suspected acute coronary syndrome who are troponin-negative. J Am Coll Cardiol. 2010; 55: 2590-8.

52. Agnello L, Bivona G, Novo G, Scazzone C, Muratore R, Levantino P, et al. Heart-type fatty acid binding protein is a sensitive biomarker for early AMI detection in troponin negative patients: a pilot study. Scand J Clin Lab Invest. 2017; 77: 428- 32

53. Bivona G, Agnello L, Bellia C, Lo Sasso B, Ciaccio M. Diagnostic and prognostic value of $\mathrm{H}-\mathrm{FABP}$ in acute coronary syndrome: Still evidence to bring. Clin Biochem. 2018; 58: 1-4. 